\title{
Changes in clinical reasoning and cognitive error after a intensive online course: A Qualitative study in medical students
}

\author{
Verónica Mir-Bezanilla ${ }^{1}$, Antonia González-Bernstein ${ }^{1}$, María-Ignacia Lüers-Sepúlveda ${ }^{1}$, \\ Rosario López-Leiva ${ }^{1}$, Joaquín Díaz-Schmidt ${ }^{1}$, Soledad Armijo ${ }^{2}$, and Cristhian \\ Pérez-Villalobos ${ }^{3}$ \\ ${ }^{1}$ Universidad del Desarrollo Facultad de Medicina Clínica Alemana \\ ${ }^{2}$ Universidad del Desarrollo Facultad de Medicina \\ ${ }^{3}$ Universidad de Concepción
}

September 28, 2020

\begin{abstract}
Introduction: Clinical reasoning is a crucial competence for medical practice and also a complex task that is susceptible to cognitive errors. It is usually taught based on whole clinical cases, from a practical rather than from a conceptual perspective. Addressing the need to improve clinical reasoning teaching into undergraduate medical curricula, it was hypothesized that medical students could benefit from a practice and theoretical approach to the clinical reasoning process. Methods: A four week online course, based on simulation and groupal reflective practice was developed, to promote metacognition between the participants. The course was delivered to 8 sixth year medical students as an elective module. A questionnaire consistent of four open-ended questions was designed to explore knowledge about clinical reasoning and cognitive errors, and was applied at the beginning and at the end of the course. A qualitative analysis was conducted with Berelson content analysis method. Results: After the course the students changed their understanding about clinical reasoning, considering it more like a process and identifying the dual nature described in modern theories of clinical decision making. Also they changed their knowledge about cognitive errors, attributing them not only to lack of knowledge, and understanding that they can actively practice some strategies to reduce cognitive bias. Discussion: This study confirmed that undergraduate students change in a positive way their concept of clinical reasoning and their knowledge about this cognitive process and cognitive errors that occur on it after a course that includes simulation and reflection.
\end{abstract}

\section{OBJECTIVE}

This research aims to describe changes in the knowledge about clinical reasoning and cognitive errors after an intensive online course based on simulation and reflective practice.

\section{METHOD}

A four-week online course, to promote active experimentation using simulation and reflection about clinical reasoning related to adult pathologies in emergency settings was developed. The course was delivered to sixth year medical students as a pilot in an elective module designed to adapt teaching to COVID19 pandemic conditions.

In order to assess changes in students' knowledge about clinical reasoning and cognitive error a qualitative study was performed using a case study research method. ${ }^{18}$

The case included eight students who were taking this course chosen by intentional sampling. Five of them were women, and all of them were taking this course for the first time. 
The course finished with a project based learning, consistent in the design and implementation of a simulation scenario and debriefing by the students organized in pairs, and a session of analysis of the implementation focused on facilitation techniques to promote reflection.

The study was approved by Universidad del Desarrollo ethical committee (48/2018, with an extension at May 2020). All volunteers participated in an informed consent procedure.

A four open-ended questions questionnaire was designed to explore knowledge about clinical reasoning, cognitive errors, the reflection process on decision making and how teamwork and communication affect decision making. It was applied at the beginning (pre-course questionnaire) and at the end of the course (post-course questionnaire).

A qualitative analysis of the responses was carried out, using Berelson's content analysis ${ }^{19}$ as a method to detect changes in the participants' perception of what clinical reasoning is and its elements. The subjects, whose privacy is reserved, are represented in Tabla 1 with Roman numerals.

\section{RESULTS}

The results of the study are presented below, organized according to each of the four used questions.

\section{What is clinical reasoning?}

In the pre-course questionnaire, the participants described the mechanism of clinical reasoning as a process or method of critical analysis, systematic reasoning or an ordered logical thought. They mentioned that in it, one deliberates at each step, focusing on clinical features of the patient. Different diagnoses, variables and ways to proceed are evaluated, using and integrating medical knowledge. Very similarly, in the post-course questionnaire, the participants conceptualized the clinical reasoning as a cognitive process of logical thinking, in which one takes the story, physical evaluation and exams of the patient, presents the differential diagnoses, analyzes the information mentioned and finally makes a decision. However, new elements were added such as the probability of illness as part of the decision process and the permanent evaluation of adopted behaviors and clinical decisions.

In both times, they acknowledged that the purpose is to face a clinical situation, develop a better decisionmaking process, determine differential diagnoses, guide exams, and then to an appropriate treatment or action. While in the post-course, they mentioned that clinical reasoning is carried out to integrate the information on a clinical case.

Regarding the context, the subjects identified that clinical reasoning is carried out in a clinical case, when facing a patient, considering their story and context both in pre and post questionnaire.

\section{What are cognitive errors?}

In the pre-course questionnaire, several participants stated that they did not know or were not sure about the concept of cognitive errors. Even though, some referred to them as mistakes or equivocations that are made. By the other hand, in the post-course questionnaire, all the participants gave a definition. Concepts like problems, traps or improvable approaches were added to the characterization of cognitive errors.

Regarding the mechanisms for these errors, in the first instance, it is described as the misinterpretation of the information or way of proceeding. Between the causes, they alluded it to the lack of knowledge, erroneous conceptions, or being pigeonholed in a diagnosis. In the post-course survey, the participants described different and multiple ways in which cognitive errors can be generated during the thinking process. It was described that the errors can occur at different stages of the process. Knowledge errors can occur because of a lack of it or mistakes in the concepts. Other influences such as personal experience, previous knowledge or beliefs are incorporated. They also refer that cognitive errors can be generated by the incorrect handling of the data, considering the process of collecting, integrating, confirming and interpreting the information. Some theoretical topics like system one, system two and cognitive bias that alters the reasoning process are also mentioned. 
By contextualizing cognitive errors, in both questionnaires, they placed them within the process of clinical reasoning.

Finally, in the second questionnaire, the idea of "consequences of cognitive errors" was incorporated. They describe that they "influence, alter or hinder clinical reasoning, so they can lead to erroneous conclusions."

\section{What do you understand from "reflection processes in clinical decision-making"?}

In both moments, most of the participants conceptualized the reflection processes in clinical decision-making, as "instances", "pauses" and "moments". Regarding the mechanism of these reflection processes, in both surveys is referred as a process in which important aspects are identified, reflected, an analysis is performed and "retrospective counting" is carried out. In the second questionnaire, participants use more complex expressions, including some theoretical concepts as the integration and confirmation of data. Also, analysis of decisions, errors and successes are added to the process.

The participants stated that the content that is analyzed is the information collected in a case, the reasoning, the actions taken and the decisions.

When describing the context of the reflection processes in clinical decision-making, in the first instance they refer to the clinical reasoning process. In the second opportunity it is specified that it is carried out before, during and after making decisions related to a clinical case.

With regard to the purpose of the reflection process in clinical decision-making, on both occasions they mention that it seeks to analyze the clinical case, for the most appropriate diagnosis and management of the patient. After the learning experience on clinical reasoning, they added that it also seeks to "it allows to evaluate the actions that were carried out and what could be improved" and "to be able to discern if this could have been done better in another way", for example.

\section{How do team-work and communication affect decision-making?}

In both surveys, the participants identified teamwork and communication as important elements in decisionmaking and referred that they affect positively in the reasoning process. In the pre-course questionnaire the participants highlight that it is important because it allows to analyze more than one perspective and to delegate tasks within the team. In the post-course questionnaire the participants recognize that teamwork and communication helps each other to feel supported and to complement each other's points of view, this way the discussion is enriched in order to consider the better option for the patient. Regarding the mechanism of teamwork, in both moments the participants highlight the importance of adequate communication and knowledge of the role of each member. In the first questionnaire it is emphasized that teamwork influences the analysis method, and the correction and feedback for the team when confronting a clinical scenario. In the second questionnaire, the evolution of teamwork is deeper described. They mention that "it works as a cognitive support for the physician that makes the diagnosis" and identified that "a good teamwork, in which everyone contributes according to their role and experience and that communicate with each other appropriately and also with patients". As to the purpose of teamwork and communication in decision making, in the first instance they indicate that it aims to be able to handle all the possible information, see and incorporate new perspectives and ways of reasoning. After the learning experience, they add that it seeks to enrich the discussion, identify and reduce biases and errors. In both instances they agree that it allows the best management for the patient. Finally, concerning the context of decision making and teamwork, in

both moments, they mention that it occurs when making decisions in a clinical setting. In the post-course questionnaire, they included when carrying out an intervention.

\section{DISCUSSION}

\section{Clinical Reasoning}

The concept of clinical reasoning described by the participants, especially after the intervention, is very similar to the concept found in literature, probably because of clinical reasoning is a mainstay in medical 
training, a necessary cognitive process to analyze and solve a patient's clinical problem, ${ }^{2}$ in an appropriate way, in the patient's benefit. ${ }^{1}$

Before the course participants express ideas that seem to reflect an understanding of clinical reasoning as a linear process and after the course they use expressions that reveal an understanding of clinical reasoning as a cyclical process, with control mechanisms that articulate and constitute a kind of continuous improvement process. ${ }^{4}$

In regards to the purpose of clinical reasoning, participants agreed that it is carried out to make a diagnosis and determine or guide towards an appropriate treatment, and as a final objective "solve a case or clinical problem". Comparing both moments, in the second one "integrating information of a clinical case" is added as part of the purpose of reasoning, and not only achieving the answer. This is consistent with the current literature, since it has been described that the most important part of the teaching of clinical reasoning emphasizes the mental process by which thinking is organized, rather than on the result alone. ${ }^{1,11}$

Regarding the context, in both instances they refer to it as a case or clinical scenario, when facing a patient. The way in which contextual factors impact the clinical reasoning performance of medical students has been studied. The importance of context in clinical reasoning is supported by the finding of a specific context, which could cause, for example, that a doctor reaches two different diagnoses having two patients with the same symptoms, findings and apparently the same diagnosis. ${ }^{10}$ Awareness of contextual factors, which can lead to cognitive errors, appears with a more comprehensive logic in the post-course. In fact, the first response is more abstract and the second is more situated and linked to the clinical task, which can help prevent or at least be attentive to this type of situation.

\section{Cognitive errors}

After the course there was an important change in the knowledge of cognitive errors and their implications. The students recognize them as "errors and mistakes", but concepts such as "traps" and "improvable approaches" were added and placed in the thought process, in a similar way that Schnapp defined cognitive errors. ${ }^{9}$ They also associate the occurrence of cognitive errors to failures in the use of system one and two, as has been described by Kahnemann ${ }^{4}$ and Norman. ${ }^{20}$

At the same time, it is described that these errors can be of various forms, and at different stages of the process. With regard to knowledge, in addition to lack of it or errors, external influences such as personal experience, previous knowledge or beliefs are incorporated. They also refer that it may imply incorrect handling of the data, both when considering, collecting, integrating, confirming or interpreting them, as was described by Kassirer et al. ${ }^{21}$ This also shows a more process-oriented view of cognitive errors rather than a mechanical consequence of lack of knowledge.

The participants mention cognitive biases, which can also alter the reasoning process. They use expressions as "shortcuts" that have been used in literature as a reference to cognitive errors. ${ }^{22}$ Heuristic processes are inherent to the human mind, and interrupt the logical line of reasoning gathering errors. ${ }^{23} \mathrm{~A}$ study that describes the most frequent heuristic errors in medical students, recognizes that there are no differences between the errors observed among third- and sixth-year students, despite the difference in knowledge that exists between students at both levels. ${ }^{24}$ In that study the students did not know the concepts of error or heuristics.

They contextualize cognitive errors within the process of clinical reasoning and identify some of the errors defined in literature. ${ }^{17}$ After the course they refer to errors as circumstances in the thought process, rather than in the final result of this. The different opinions expressed by the participants about cognitive errors reflect the individual nature of the clinical reasoning process and how we are affected in the way we make decisions according to our own context. ${ }^{10}$

\section{Reflection processes in clinical decision-making}

When conceptualizing the reflection processes in decision making, in both surveys the participants define 
it as "instances", "pauses", and "moments". These expressions could represent a need for calm, to get away to stop and think, or to protect themselves to think. It is possible that for students facing complex situations is associated with a high cognitive load that prevents them from performing a thorough analysis of their actions, since they are busy in the process of collecting and processing information. Prior to process automation, students invest a lot of attention, focus, and effort in decision making, so a student may demand "time out" to learn how to do something that he or she can then do "during" clinical care.

Perhaps many of the tasks are assumed as routine, as part of the method of inquiry and not as a thought process. Only after the learning experience about clinical reasoning, they considered that the reflection process is an inherent part of clinical decision making and an ability that allows them to evaluate the measures that were taken and what could be improved" or to "be able to discern whether it could have been done in a better way, for example". These expressions are usual in the debriefing process associated with high fidelity simulation, ${ }^{17}$ and maybe our experience reflecting on the actions performed at simulation scenarios contributes to this learning process.

\section{Team-work and communication to improve decision-making}

According to the literature, teamwork is indispensable to carry out an adequate management when facing a patient. This is because it allows the exchange of information and knowledge with the team, which is crucial for clinical reasoning. ${ }^{25}$ In this study, participants referred to the relevance of teamwork in the same way, saying that it allows more perspectives and points of view to be analyzed, but moves deeply on their reflections, when they recognized that "we have a tendency to be closed in on our beliefs and knowledge, letting go of different perspectives that can often consider better options for a patient". This expression recognizes that teamwork is a good tool to avoid some kind of cognitive errors like anchorage or premature closure, ${ }^{17}$ by example.

Some of the ideas that the participants identify regarding teamwork, includes leadership, peer support, mutual performance monitoring, three of the five dimensions proposed by Salas ${ }^{26}$ as an effective teamwork. The literature also highlights the importance of having a "shared mental model" among team members, mainly through the exchange of information among them, ${ }^{27}$ an idea that was also recognized in our study.

The participants in this study consider that teamwork is beneficial for team-members and for the patients. Effective teamwork and good communication between members reduces errors in clinical practice, increases safety for patients and delivers effective treatment. ${ }^{28}$

\section{CONCLUSSION}

This study shows that undergraduate students change their concept of clinical reasoning and their knowledge about this cognitive process and cognitive errors that occur on it after a course that includes simulation and reflection.

Considering that medical decisions can be influenced by factors beyond the logic of the thought process, we believe that it is useful for future professionals to be aware of the existence of these processes and elements so that they can be aware of how they affect their performance in clinical practice and can develop strategies to regulate their impact. .

We recommend incorporating the teaching of clinical reasoning and cognitive or heuristic errors as part of the medical curriculum.

\section{ACKNOWLEDGMENTS}

The authors thank Dr. Claudia Behrens for providing feedback on earlier versions of the manuscript that helped shape the final product. Also thank Dr. Marcela Castillo, School of Medicine Director at Universidad del Desarrollo, for supporting our efforts in designing and implementing innovative teaching strategies.

\section{REFERENCES}


1. Silva V, Mccoll P, Pérez C, Searle M, Goset J. Instrumento para el desarrollo del razonamiento clínico [Development of an instrument to assess clinical reasoning]. Rev Med Chil. 2018;146(12):1466-1470. doi:10.4067/s0034-98872018001201466

2. Losada Guerra Jorge Luis, Hernández Navarro Elena V., Salvat Quesada Miguel, Losada Hernández José Omar. Evolución histórica del razonamiento clínico. MEDISAN [Internet]. 2019 Dic [citado 2020 Sep 19] ; 23( 6 ): 1164-1180. Disponible en: http://scielo.sld.cu/scielo.php?script=sci_arttext\&pid=S1029-30192019000601164\&lng=es. Epub 20-Dic-2019.

3. ten Cate, O., \& Durning, S. J. Understanding Clinical Reasoning from Multiple Perspectives: A Conceptual and Theoretical Overview. In O. ten Cate (Eds.) et. al., Principles and Practice of Case-based Clinical Reasoning Education: A Method for Preclinical Students. Springer. 2017.(pp. 35-46).

4. Kahneman, D. Thinking, fast and slow. New York: Farrar, Straus and Giroux. 2011. 485p.

5. Croskerry P, Petrie DA, Reilly JB, Tait G. Deciding about fast and slow decisions. Acad Med. 2014;89(2):197-200. doi:10.1097/ACM.0000000000000121.

6. Custers EJ. Medical education and cognitive continuum theory: an alternative perspective on medical problem solving and clinical reasoning. Acad Med. 2013;88(8):1074-1080. doi:10.1097/ACM.0b013e31829a3b10

7. Norman GR, Eva KW. Diagnostic error and clinical reasoning. Med Educ. 2010;44(1):94-100. doi:10.1111/j.1365-2923.2009.03507.x

8. Graber M, Gordon R, Franklin N. Reducing diagnostic errors in medicine: what's the goal?. Acad Med. 2002;77(10):981-992. doi:10.1097/00001888-200210000-00009

9. Schnapp BH, Sun JE, Kim JL, Strayer RJ, Shah KH. Cognitive error in an academic emergency department. Diagnosis (Berl). 2018;5(3):135-142. doi:10.1515/dx-2018-0011

10. McBee E, Ratcliffe T, Schuwirth L, et al. Context and clinical reasoning: Understanding the medical student perspective. Perspect Med Educ. 2018;7(4):256-263. doi:10.1007/s40037-018-0417-x

11. Montaldo L G, Herskovic L P. Aprendizaje del razonamiento clínico por reconocimiento de patrón, en seminarios de casos clínicos prototipos, por estudiantes de tercer año de medicina [Teaching of clinical reasoning to medical students using prototypical clinical cases]. Rev Med Chil. 2013;141(7):823-830. doi:10.4067/S0034-98872013000700001

12. Manesh R, Dhaliwal G. Digital Tools to Enhance Clinical Reasoning. Med Clin North Am. 2018;102(3):559-565. doi:10.1016/j.mcna.2017.12.015

13. Bae J, Lee J, Jang Y, Lee Y. Development of simulation education debriefing protocol with faculty guide for enhancement clinical reasoning. BMC Med Educ. 2019;19(1):197. Published 2019 Jun 11. doi:10.1186/s12909-019-1633-8

14. Chon SH, Timmermann F, Dratsch T, et al. Serious Games in Surgical Medical Education: A Virtual Emergency Department as a Tool for Teaching Clinical Reasoning to Medical Students. JMIR Serious Games . 2019;7(1):e13028. Published 2019 Mar 5. doi:10.2196/13028

15. Surry LT, Torre D, Trowbridge RL, Durning SJ. A mixed-methods exploration of cognitive dispositions to respond and clinical reasoning errors with multiple choice questions. BMC Med Educ . 2018;18(1):277. Published 2018 Nov 23. doi:10.1186/s12909-018-1372-2

16. Bhatti A. Cognitive bias in clinical practice - nurturing healthy skepticism among medical students. Adv Med Educ Pract . 2018;9:235-237. Published 2018 Apr 10. doi:10.2147/AMEP.S149558

17. Pennaforte T, Moussa A, Loye N, Charlin B, Audétat MC. Exploring a New Simulation Approach to Improve Clinical Reasoning Teaching and Assessment: Randomized Trial Protocol. JMIR Res Protoc . 2016;5(1):e26. Published 2016 Feb 17. doi:10.2196/resprot.4938

18. Stake, R. E. Multiple case study analysis. New York, Guilford press, 2013. p342

19. Marris, P., Thornham, Sue. Media Studies: A reader 2nd edition. New York, New York University Press, 2000, p 896.

20. Norman GR, Monteiro SD, Sherbino J, Ilgen JS, Schmidt HG, Mamede S. The Causes of Errors in Clinical Reasoning: Cognitive Biases, Knowledge Deficits, and Dual Process Thinking. Acad Med . 2017;92(1):23-30. doi:10.1097/ACM.0000000000001421

21. Kassirer JP, Kopelman RI. Cognitive errors in diagnosis: instantiation, classification, and consequences. 
Am J Med . 1989;86(4):433-441. doi:10.1016/0002-9343(89)90342-2

22. Redelmeier DA. Improving patient care. The cognitive psychology of missed diagnoses. Ann Intern Med . 2005;142(2):115-120. doi:10.7326/0003-4819-142-2-200501180-00010

23. Crupi V, Elia F. Understanding and improving decisions in clinical medicine (I): Reasoning, heuristics, and error. Intern Emerg Med. 2017 Aug;12(5):689-691. doi: 10.1007/s11739-017-1665-1. Epub 2017 Apr 21. PMID: 28432543.

24. Rylander M, Guerrasio J. Heuristic errors in clinical reasoning. Clin Teach. 2016 Aug;13(4):287-90. doi: 10.1111/tct.12444. Epub 2015 Sep 23. PMID: 26395611.

25. Fürstenberg S, Oubaid V, Berberat PO, Kadmon M, Harendza S. Medical knowledge and teamwork predict the quality of case summary statements as an indicator of clinical reasoning in undergraduate medical students. GMS J Med Educ . 2019;36(6):Doc83. Published 2019 Nov 15. doi:10.3205/zma001291

26. Salas E, Sims DE, Burke CS. Is there a "Big Five" in Teamwork? Small Group Research . 2005;36(5):555599. doi:10.1177/1046496405277134

27. Weller J, Boyd M, Cumin D. Teams, tribes and patient safety: overcoming barriers to effective teamwork in healthcare.Postgrad Med J . 2014;90(1061):149-154. doi:10.1136/postgradmedj-2012-131168

28. Gluyas H. Effective communication and teamwork promotes patient safety. Nurs Stand . 2015;29(49):5057. doi:10.7748/ns.29.49.50.e10042

Table 1: Pre and post intervention quotes about clinical reasoning

\begin{tabular}{ll}
\hline Question & Concept \\
What is clinical & Mechanism of clinical \\
reasoning? & reasoning
\end{tabular}

Purpose of clinical reasoning

Context of clinical reasoning

\section{Pre-course questionnaire quotes}

Basically, it is reasoning about the symptoms and signs of a sick patient. (III) It is the method through which different diagnoses are evaluated $(\mathrm{V})$ ordering of logical thinking (I) It is a process of critical analysis (VI) Using and integrating knowledge in medicine (VIII)

To face a clinical situation (I) To [...] be able to do a better decision-making (VII) to reach a possible diagnosis (VII) to solve clinical cases. (VIII)

when facing a patient (II) carrying out a diagnosis (IV)

\section{Post-course questionnaire quotes} Logical thinking process (I) Process by which decision-making is carried out (II) It is about [...] raising different differential diagnoses, some of which are more likely than others (III) cognitive process that allows the evaluation of adopted behaviors and management (VI) To identify different factors that suggest a diagnosis (I) To integrate the information of a clinical case to achieve a diagnosis and $[\ldots]$ a correct treatment (VIII) that applies to a clinical case (I) when approaching to a patient (II) considering the history and the context of the patient (III 1B) 
What are cognitive errors?
Definition of cognitive errors

Mechanism and causes of cognitive errors

Context of cognitive errors

Consequence of cognitive errors
I don't know (IV, V) To make a mistake(VIII)

Misinterpret (I)

Misinterpret the information from different sources (III) To pigeonhole in just one diagnosis (VI) Because of the lack of medical knowledge (VII)

when [...] making decisions (II) either in terms of exam request, (VI) either in [...] procedures, (VI)
They are problems in the process (I) Mistakes that are commited (II) They are improbable approaches to a patient (V) Pitfalls in the thinking process (VII) They are systematic mistakes (VIII) that influence clinical reasoning, "trapping" it, (IV) that make us change reasoning in some way (IV) the causes of this errors can have MULTIPLE reasons, many times they are influenced by personal experience of one person, for example (III) beliefs (IV) because of a mishandling of the data (either it's gathering, integration and/or confirmation )(VI) either because of lack of knowledge either because of $[\ldots]$ considering wrongly some of the data (I) and cognitive biases (II) (they can belong to system 1 or 2) (V) That can be because of lack of or wrong knowledge, (VII) That occur when there is a bias that affects the reasoning process (VIII)

that are made at the time of carrying out clinical reasoning. (II) that occur in particular situations $(\mathrm{V})$ in the thinking process (VII) That may lead to wrong conclusions (I) because they alter the way in which we think (IV) that hinder an appropriate clinical reasoning (VI 2B) 
What do you understand from "reflection processes in clinical decision-making"?
Definition of reflection processes in clinical decision-making

Mechanism of reflection processes in clinical decision-making

Content of reflection processes in clinical decision-making

Context of reflection processes in clinical decision-making instances (VII) moments (V) pauses one takes (VI) "check points" (II)

process of connecting the dots (I) making a retrospective count (VIII) self-analysis moments (V)
Pauses and moments (I,II,V) They are instances or spaces (VI) (in this) gaps of time (VI) "protected" time (VII) Process (VIII) in which time is given to think about the decisions that are made in the clinical reasoning process(II) the performance is analyzed in a reflexive way $(\mathrm{V})$ analyzing rigorously the conducts that have been taken until now, that are being taken and/or planned to take (VI) one should be able to question his/her own actions [...] identify errors and hints (VI) analysis the obtained data, integrated and confirm them (VII) Assess retrospectively the reasoning that was made, the decisions made in relation to it (VIII)

about a patient's clinical the decisions that are case (III) considering the patient's history, reason for consultation, context and current situation (VI) of my [...] reasoning to reach a decision (VIII)

in the process of clinical reasoning (IV) made in the process of clinical reasoning (II)

Reflect about the patient's case, exams, differential diagnoses (III) the reasoning carried out and the decisions made in relation to it. (VIII) the performance $(\mathrm{V})$ their actions, [...] mistakes and successes, (VI) prior to decision making, (VI) that occurs during the clinical case (I) when making a decision and after making it (IV) 
Purpose of reflection processes in clinical decision-making

How do team-work and communication affect decision-making?
Relevance of team-work and communication in decision making to reach a conclusion (I) to think and guide us to an etiology (III) to analyze [...] the diagnostic variables and to appraise the best decision for the patient (VII)

it's a key factor (I) affects in a positive way (VI) it is essential to have good communication in the healthcare team (VIII) to be able to evaluate all the information obtained. (I) see which treatment is appropriate for the patient or if any treatment is necessary. (III) in order to reach the most accurate diagnosis and thus the appropriate treatment (VII) It allows to evaluate the actions that were carried out and what could be improved (II) to be able to discern if it could have been done better in another way (IV) to define if it is the most appropriate (management) according to the patients and their context. (VI) and determine if it is helpful or if a change in behavior is required. (VI)

it's a key factor (I) very important (III) affects in a very positive way (VI) Good teamwork is essential within the healthcare team. (VIII) 
Why is team-work and communication important in decision-making?

Mechanism in which team-work and communication affect decision-making because it allows us to delegate tasks,] to have different points of view (II) Because it can lead us towards a broader differential diagnosis, it can open us to [...] different points of view different treatment options (III) because we can broaden the analysis (VI) because we can [...] accept other points of view (VI) Helps to see the perspective of others (IV) helps to [...] incorporate other ways of reasoning to ours (IV)

feedback of the team $(\mathrm{V})$ it must be a clear and precise communication (VII) it must be confidence in the team (VII) must know the roles of each part of the team. (VII) make one feel more supported (V) One always need a team to lean on (I) We always need [...] to support each other (I) Allows us to make decisions in a more confident way. (II) Allows to complement each other (II) Because they help to "get out from your own thoughts"" (III) Because they help to [...] listen to others (III) The discussion is enriched if these (other's perspectives) are integrated. (IV) Mainly because we have a tendency to lock in our beliefs and knowledge, letting out different perspectives, that can be better for the patient.(VI)

Complement the ways of thinking and take dimensions that you didn't consider before (V) It works as a cognitive support for the physician that makes the diagnosis (VIII) A good teamwork, in which everyone contributes according to their role and experience and that communicate with each other appropriately and also with patients (VII) 
Purpose of team-work and communication in decision-making

Context in which team-work and communication affect decision-making to handle all the information correctly (I) Helps to consider and incorporate the perspective of others and other forms of reasoning to ours (IV) To achieve good performance. (V)

To make better decisions for the patient (VII)

Benefit for its members (healthcare team) and patients (VIII)

when dealing with a patient (II) when making decisions (VII)
The discussion is enriched if these (other's opinions) are integrated (IV) in order to make the best decision for the patient and reduces bias and errors (II) It also allows to learn from each other's reasoning process (IV) It allows us to identify mistakes that we could not have realized by ourselves, or confirm that what you are doing is appropriate. (VI) It favors a more effective and favorable management for the patient. (VI) it causes fewer errors in the process of making decisions (VII) It will make the process better (VII) lightening the burden of each member (of the team) (VIII) for the patient (II, III, VI) about the clinical case (III) when we are making a decision. (IV, V, VII) at the time of an intervention, (VIII) 